\title{
Gadolinium Retention in the Brain: An MRI Relaxometry Study of Linear and Macrocyclic Gadolinium-Based Contrast Agents in Multiple Sclerosis
}

\author{
(D) Y. Forslin, (D). Martola, (D) A. Bergendal, (D). Fredrikson, (D) M.K. Wiberg, and (D)T. Granberg
}

\begin{abstract}
BACKGROUND AND PURPOSE: Brain gadolinium retention is consistently reported for linear gadolinium-based contrast agents, while the results for macrocyclics are contradictory and potential clinical manifestations remain controversial. Furthermore, most previous studies are based on conventional T1-weighted MR imaging. We therefore aimed to quantitatively investigate longitudinal and transversal relaxation in the brain in relation to previous gadolinium-based contrast agent administration and explore associations with disability in multiple sclerosis.
\end{abstract}

MATERIALS AND METHODS: Eighty-five patients with MS and 21 healthy controls underwent longitudinal and transverse relaxation rate $\left(R_{1}\right.$ and $\left.R_{2}\right)$ relaxometry. Patients were divided into linear, mixed, and macrocyclic groups based on previous gadolinium-based contrast agent administration. Neuropsychological testing was performed in 53 patients. The dentate nucleus, globus pallidus, caudate nucleus, and thalamus were manually segmented. Repeatability measures were also performed.

RESULTS: The relaxometry was robust (2.0\% scan-rescan difference) and detected higher $R_{1}$ (dentate nucleus, globus pallidus, caudate nucleus, thalamus) and $R_{2}$ (globus pallidus, caudate nucleus) in patients receiving linear gadolinium-based contrast agents compared with controls. The number of linear gadolinium-based contrast agent administrations was associated with higher $R_{1}$ and $R_{2}$ in all regions (except $R_{2}$ in the thalamus). No similar differences and associations were found for the macrocyclic group. Higher relaxation was associated with lower information-processing speed (dentate nucleus, thalamus) and verbal fluency (caudate nucleus, thalamus). No associations were found with physical disability or fatigue.

CONCLUSIONS: Previous linear, but not macrocyclic, gadolinium-based contrast agent administration is associated with higher relaxation rates in a dose-dependent manner. Higher relaxation in some regions is associated with cognitive impairment but not physical disability or fatigue in MS. The findings should be interpreted with care but encourage studies into gadolinium retention and cognition.

ABBREVIATIONS: $\mathrm{CN}=$ caudate nucleus; $\mathrm{DN}=$ dentate nucleus; $\mathrm{EDSS}=$ Expanded Disability Status Scale; $\mathrm{GBCA}=$ gadolinium-based contrast agent; $\mathrm{GP}=$ globus pallidus; $R_{1}=$ longitudinal relaxation rate; $R_{2}=$ transverse relaxation rate; $S D M T=$ Symbol Digit Modalities Test

B rain gadolinium retention after administration of gadoliniumbased contrast agents (GBCAs) has been demonstrated, mainly for linear GBCAs in the dentate nucleus (DN) and globus pallidus (GP), by several studies since the initial report in $2014 .^{1}$ Studies of macrocyclic GBCAs, however, remain contradictory, with several studies showing no lasting T1 hyperintensities, ${ }^{2-4}$

Received March 19, 2019; accepted after revision May 20.

From the Department of Clinical Neuroscience (Y.F., J.M., A.B., S.F., M.K.W., T.G.), Karolinska Institutet, Stockholm, Sweden; Departments of Radiology (Y.F., J.M., M.K.W., T.G.) and Neurology (S.F.), Karolinska University Hospital, Stockholm, Sweden; and Department of Medical and Health Sciences (M.K.W.), Division of Radiological Sciences, Linköping University, Linköping, Sweden.

This research was supported by Karolinska Institutet and the Stockholm County Council through an ALF grant.

Please address correspondence to Yngve Forslin, MD, Department of Radiology, Cl-46, Karolinska University Hospital, 14186 Stockholm, Sweden; e-mail: yngve.forslin@ki.se while a few retrospective studies had positive findings, ${ }^{5-7}$ though there has been criticism regarding some of these results. ${ }^{8}$ Animal studies have, however, found brain gadolinium retention after multiple administrations of macrocyclic GBCAs, though to a lesser extent than for linear GBCAs. ${ }^{2}$ This finding suggests that detecting $\mathrm{T} 1$ alterations after macrocyclic GBCAs may require more sensitive methods than conventional MR imaging. Furthermore, the semiquantitative approach of signal intensity ratios based on T1-weighted imaging may also be flawed by the need for scaling the arbitrary signal intensities with a reference region. Histopathologic studies have shown that gadolinium retention is widespread and also occurs in brain regions that are often used as

Indicates article with supplemental on-line tables.

Indicates article with supplemental on-line photos.

http://dx.doi.org/10.3174/ajnr.A6112 
Table 1: Demographics of the study population and the number of GBCA administrations

\begin{tabular}{|c|c|c|c|c|}
\hline & $\begin{array}{l}\text { Healthy } \\
\text { Controls }\end{array}$ & Linear MS Group & Mixed MS Group & Macrocyclic MS Group \\
\hline Participants (No.) & 21 & 11 & 59 & 15 \\
\hline Male/female ratio & 10:11 & $3: 8$ & $18: 41$ & 4:11 \\
\hline Age (mean) (yr) & $35.9 \pm 13.8$ & $51.0 \pm 10.7$ & $43.8 \pm 10.3$ & $37.4 \pm 13.7$ \\
\hline MS duration (mean) (yr) & NA & $19.8 \pm 9.3$ & $12.9 \pm 8.1$ & $5.1 \pm 6.0$ \\
\hline MS subtype, RR/SP/PP & NA & $5 / 6 / 0$ & $39 / 17 / 3$ & $14 / 1 / 0$ \\
\hline EDSS score (median) (range) & NA & $2.5(0-6) ; n=11$ & $2(08.5) ; n=54$ & $2(1-4) ; n=13$ \\
\hline SDMT score (median) (range) & NA & $-0.19(-1.47-0.84) ; n=5$ & $-1.1(-3.9-1.4) ; n=36$ & $-1.3(-4.3-0.51) ; n=12$ \\
\hline Verbal fluency test score (median) (range) & NA & $0.74(-1.82-.36) ; n=4$ & $-0.24(-2.47-2.17) ; n=17$ & $-1.32(-2.94-.04) ; n=6$ \\
\hline $\begin{array}{l}\text { MS lesion volume (median } \pm \text { interquartile } \\
\text { range) }(\mathrm{mL})\end{array}$ & NA & $5.1 \pm 10.1$ & $3.1 \pm 9.2$ & $2.4 \pm 4.2$ \\
\hline Brain parenchymal fraction (\%) & $88.0 \pm 2.4$ & $79.6 \pm 4.9$ & $82.7 \pm 5.9$ & $84.6 \pm 5.0$ \\
\hline $\begin{array}{l}\text { Linear GBCA administrations (No.) (median) } \\
\text { (range) }\end{array}$ & 0 & $3(1-7)$ & 4 (1-19) & $0(0)$ \\
\hline $\begin{array}{l}\text { Macrocyclic GBCA administrations (No.) } \\
\text { (median) (range) }\end{array}$ & 0 & 0 & $2(1-4)$ & $3(1-6)$ \\
\hline $\begin{array}{l}\text { Time since last GBCA administration } \\
\text { (median) (range) (mo) }\end{array}$ & NA & $52(24-172)$ & 12 (3-91) & 7 (3-14) \\
\hline
\end{tabular}

Note:-PP indicates primary-progressive; RR, relapsing-remitting; SP, secondary-progressive; NA, not applicable.

${ }^{a}$ Numbers are given as means unless otherwise specified.

a reference, such as the thalami. ${ }^{9}$ Relaxometry provides the means to study gadolinium retention quantitatively without the need for a reference region, but so far, there are only a few T1-relaxometry studies in the context of gadolinium retention, ${ }^{10,11}$ and no T2relaxometry studies. While chelated GBCAs lead to a shortening of T1, they also cause a shortening of T2. ${ }^{12}$

The clinical significance of the retained gadolinium is debated, but medical authorities have used the precaution of moderating the use of mainly the linear GBCAs. ${ }^{13,14}$ A large retrospective study of patients undergoing contrast-enhanced MR imaging without a neurologic indication did not find any increased incidence of Parkinsonism in the exposed patients. ${ }^{15}$ Furthermore, a preliminary report from an aging cohort did not show any cognitive decline or worsened motor performance in those who were exposed to GBCAs during the study. ${ }^{16}$ However, in a small retrospective long-term follow-up study in multiple sclerosis, there were associations between $\mathrm{T} 1$ signal intensity ratio increases and impaired cognitive function, prompting further studies on the subject. ${ }^{17}$

We aimed to apply a new robust method for simultaneous T1 and $\mathrm{T} 2$ relaxometry in a prospective cohort of patients with MS and healthy controls to investigate associations between both longitudinal and transverse relaxation rates in the DN, GP, caudate nucleus $(\mathrm{CN})$, and thalamus with exposure to linear and macrocyclic GBCAs. We also aimed to study possible associations between longitudinal and transverse relaxation rate $\left(R_{1}\right.$ and $\left.R_{2}\right)$ values and cognitive and physical disability scores in MS.

\section{MATERIALS AND METHODS \\ Study Population}

This prospective cross-sectional cohort study was approved by the local ethics review board, and written informed consent was obtained from all participants. We consecutively recruited patients with MS referred for brain MR imaging between January and June 2015 at Karolinska University Hospital in Huddinge, Stockholm, Sweden. The only inclusion criterion was an MS diagnosis according to the concurrent $2010 \mathrm{McDonald}$ criteria for patients. Exclu- sion criteria were neurologic diseases (other than MS for patients), traumatic brain injury, or imaging artifacts. Additionally, 23 age- and sex-matched healthy controls without exposure to GBCAs were recruited. In total, 88 patients with MS consented to participate, but 2 were excluded due to previous brain trauma and 1 due to movement artifacts. Among the controls, 2 were excluded due to widespread white matter hyperintensities and a cerebellar cardiovascular lesion in 1 of the 2 excluded controls. In total, 85 patients with MS and 21 healthy controls were found eligible and were enrolled in the study. The demographics of the study population is detailed in Table 1 .

The 85 patients with MS were divided into 3 groups depending on which type of GBCA they had received. The linear group consisted of 11 patients who had previously received only linear GBCAs, nonionic gadodiamide (Omniscan; GE Healthcare, Piscataway, New Jersey), and/or linear ionic gadopentetate dimeglumine (Magnevist; Bayer HealthCare Pharmaceuticals, Wayne, New Jersey). The mixed group consisted of 59 patients who had received linear nonionic gadodiamide (Omniscan) and/or linear ionic gadopentetate dimeglumine (Magnevist) as well as macrocyclic gadoterate meglumine (Dotarem; Guerbet, Aulnay-sousBois, France). The macrocyclic group consisted of 15 patients who had received only gadoterate meglumine (Dotarem). None of the 21 healthy controls had received any GBCAs.

To evaluate the repeatability of the relaxometry MR imaging scans, 14 patients with MS and 17 healthy controls also underwent a second MR imaging acquisition with repositioning immediately after the first scan. The demographics of the repeatability cohort is presented in On-line Table 1.

\section{Image Acquisition}

All imaging was performed on the same 3T Magnetom Trio MR imaging scanner (Siemens, Erlangen, Germany) with a 12-channel head coil. In addition to the national Swedish brain MR imaging protocol for MS, ${ }^{18}$ a quantitative MR imaging sequence for simultaneous $\mathrm{T} 1$ and $\mathrm{T} 2$ relaxometry was applied without administration of any GBCA. The quantitative sequence consisted of a saturation-recovery TSE sequence with 2 TEs and 4 TRs, for 
which the acquisition order was changed with each repetition, resulting in 4 different TIs. Both the phase and magnitude data were saved, providing a total of 16 images per voxel. A least squares fit was applied to the data to obtain the $R_{1}$ and $R_{2}\left(R_{1}=1 /\right.$ $\mathrm{T} 1$ and $\mathrm{R}_{2}=1 / \mathrm{T} 2$, with a unit of second ${ }^{-1}$ ) in the SyMRI software 11.0 Beta 4 for Mac (Synthetic MR, Linköping, Sweden). ${ }^{19}$ The technique has been validated as having good accuracy for T1 and $\mathrm{T} 2$ quantifications. ${ }^{20}$ The quantitative maps were also used to synthesize T1- and T2-weighted images on the basis of the default settings in the same software. SyMRI was further used to measure the brain parenchymal fraction (brain parenchymal volume normalized to the intracranial volume) as a measure of brain atrophy, which has been shown to be a robust atrophy measure in MS. ${ }^{21,22}$ For the patients with MS, the lesion-probability algorithm in the Lesion Segmentation Toolbox 2.0.12 (Technische Universität München, Munich, Germany) for SPM12 (http://www.fil.ion.u-

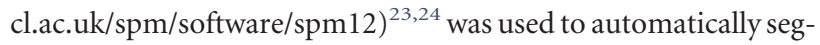
ment MS lesions on the basis of a 3D-FLAIR acquisition. The lesion masks were manually corrected by a resident in radiology (T.G.) to obtain the MS lesion volumes. The MR imaging parameters are detailed in On-line Table 2.

\section{Radiologic Evaluation}

On the basis of previous publications, ${ }^{3,9,25}$ the DN, GP, head of the $\mathrm{CN}$, and thalamus were a priori selected as ROIs. The segmentations were performed by a resident in radiology (Y.F.) in ITK-SNAP, Version 3.4.0 (www.itksnap.org) ${ }^{26}$ on the synthetic T1- and T2-weighted images. As exemplified in On-line Fig 1, synthetic T2-weighted images were chosen to easily identify the DN, and synthetic T1-weighted images, to identify the CN, GP, and thalamus. The outer edges of the structures were not included in the segmentations to mitigate partial volume effects. Furthermore, lesioned voxels were excluded from the ROI. On-line Fig 2 illustrates how lacunar infarcts within the caudate nucleus were avoided. The segmentations were applied to the inherently aligned $R_{1}$ and $R_{2}$ maps using FMRIB Software Library tools, Version 6.0 (http://fsl.fmrib.ox.ac.uk/fsl) to extract the relaxation values for each structure. ${ }^{27}$

\section{Clinical Information}

The radiologic and clinical charts of the patients with MS were reviewed to obtain information on the number and type of previous GBCA administrations, MS disease duration, MS subtype, and disease-modifying therapy. Charts were also used to extract disability scores from the Expanded Disability Status Scale (EDSS), Fatigue Severity Scale, Symbol Digit Modalities Test (SDMT), and verbal fluency test. The cognitive test scores were normalized to $z$ scores by adjusting for age, educational level, and sex on the basis of normative data. Only results within 6 months from the MR imaging examination were accepted for the study.

\section{Statistics}

SPSS, Version 24.0 (IBM, Armonk, New York) was used for the statistical analyses. Measures of the relaxation values from the paired right and left anatomic structures were averaged. The Shapiro-Wilk test was used to determine whether the data were normally distributed. The $\mathrm{R}_{1}$ and $\mathrm{R}_{2}$ values were normally distributed, except for the $\mathrm{R}_{2}$ values in the GP for the macrocyclic group that were positively skewed. After exclusion of 1 extreme outlier ( $>3$ interquartile ranges of the data), normal distribution was achieved for all relaxation values. The ordinal EDSS data were, as expected, positively skewed, and normal distribution was achieved after a logarithmic transformation. The scores of SDMT, verbal fluency, and the Fatigue Severity Scale were all normally distributed.

Group comparisons were performed with 1-way ANOVA, followed by the Hochberg post hoc test for pair-wise comparisons among the groups. Multiple linear regression analyses were used to investigate associations between the number of received GBCA administrations (independent variable) and the $R_{1}$ and $R_{2}$ values (dependent variable). The healthy controls were included as a baseline reference in the regression analyses for each group. To increase the generalizability of the results, we excluded 1 patient with a high (0.45) leverage value (who had received 19 GBCA administrations) from the linear regression analyses.

Associations between cognitive and physical disability scores (dependent variable) and $\mathrm{R}_{1}$ and $\mathrm{R}_{2}$ values (independent variable) were evaluated with multiple linear regression. To correct for the MS disease severity, we additionally performed these regression analyses with the disease duration, lesion volume, and brain parenchymal fraction added as covariates.

The scan-rescan repeatability was assessed by calculating the relative change in relaxation values between the 2 measurements by dividing the absolute difference by their mean: |Scan 1 - Scan 2| / Mean (Scan $1+$ Scan 2).

An $\alpha$ level of .05 was used to determine statistical significance, which was adjusted to .023 for the regression and ANOVA analyses after correction for the false discovery rate. ${ }^{28}$

\section{RESULTS}

\section{Repeatability}

The median scan-rescan difference of the $\mathrm{R}_{1}$ and $\mathrm{R}_{2}$ measurements among all regions was $2.0 \%$. The difference ranged between $0.8 \%$ and $2.1 \%$ for the DN, thalamus, and CN, while the GP had a median difference of $4.1 \%$ and $4.2 \%$, respectively, for the $\mathrm{R}_{1}$ and $\mathrm{R}_{2}$ measurements. On-line Table 3 details the repeatability for $\mathrm{R}_{1}$ and $\mathrm{R}_{2}$ in all ROIs.

\section{Group Differences}

There were overall differences in $\mathrm{R}_{1}$ and $\mathrm{R}_{2}(P<.001-.031)$ among the 4 groups (linear, mixed, macrocyclic, and controls) in all ROIs in the 1-way ANOVA, except the $\mathrm{R}_{2}$ values in the $\mathrm{DN}$ $(P=.05)$ and thalamus $(P=.66)$. The Hochberg post hoc test was used to explore between-group differences, as further detailed in Table 2. The $\mathrm{R}_{1}$ was higher in the DN, GP, and CN in both the linear and mixed groups compared with the healthy controls. The $\mathrm{R}_{1}$ was higher in the thalamus, and $\mathrm{R}_{2}$ was higher in both the GP and $\mathrm{CN}$ in the mixed group compared with the healthy controls.

\section{Associations of GBCA Administrations with $R_{\mathbf{1}}$ Values}

Table 3 details all regression analysis results between the number of GBCA administrations and relaxation rates. In the linear group, a higher number of GBCA administrations was associated 
Table 2: Group comparisons of the relaxation rates ${ }^{\mathrm{a}}$

\begin{tabular}{|c|c|c|c|c|c|c|c|c|}
\hline & \multicolumn{2}{|c|}{ Dentate Nucleus } & \multicolumn{2}{|c|}{ Globus Pallidus } & \multicolumn{2}{|c|}{ Caudate Nucleus } & \multicolumn{2}{|c|}{ Thalamus } \\
\hline & $\mathbf{R}_{1}$ & $\mathbf{R}_{\mathbf{2}}$ & $\mathrm{R}_{1}$ & $\mathbf{R}_{2}$ & $\mathbf{R}_{1}$ & $\mathbf{R}_{2}$ & $\mathrm{R}_{1}$ & $\mathbf{R}_{\mathbf{2}}$ \\
\hline Linear & $1.28 \pm 0.05$ & $16.74 \pm 1.96$ & $1.31 \pm 0.05$ & $20.52 \pm 1.74$ & $0.96 \pm 0.03$ & $14.44 \pm 0.62$ & $1.14 \pm 0.05$ & $14.45 \pm 0.54$ \\
\hline Mix & $1.29 \pm 0.05$ & $16.59 \pm 1.21$ & $1.29 \pm 0.06$ & $20.89 \pm 1.47$ & $0.95 \pm 0.04$ & $14.75 \pm 0.83$ & $1.15 \pm 0.05$ & $14.64 \pm 0.50$ \\
\hline Macrocyclic & $1.26 \pm 0.04$ & $15.87 \pm 1.14$ & $1.27 \pm 0.04$ & $20.50 \pm 1.101$ & $0.93 \pm 0.02$ & $14.18 \pm 0.74$ & $1.15 \pm 0.03$ & $14.55 \pm 0.48$ \\
\hline Controls & $1.23 \pm 0.05$ & $15.86 \pm 0.96$ & $1.24 \pm 0.06$ & $19.63 \pm 1.24$ & $0.91 \pm 0.03$ & $13.88 \pm 0.50$ & $1.12 \pm 0.04$ & $14.64 \pm 0.47$ \\
\hline Linear vs controls & .026 & .32 & $.012^{\mathrm{b}}$ & .52 & $.006^{\mathrm{b}}$ & .28 & .41 & .87 \\
\hline Mix vs controls & $>.001^{b}$ & .14 & $.004^{b}$ & $.004^{\mathrm{b}}$ & $<.001^{\mathrm{b}}$ & $<.001^{\mathrm{b}}$ & $.020^{\mathrm{b}}$ & 1.0 \\
\hline Macrocyclic vs controls & .13 & 1.0 & .55 & .41 & .69 & .82 & .25 & 1.0 \\
\hline Linear vs mix & .99 & .99 & .89 & .97 & 1.0 & .79 & 1.0 & .82 \\
\hline Linear vs Macrocyclic & .98 & .44 & .42 & 1.0 & .27 & .95 & 1.0 & 1.0 \\
\hline Mix vs macrocyclic & .64 & .32 & .77 & .93 & .18 & .07 & 1.0 & .99 \\
\hline
\end{tabular}

${ }^{a}$ The upper 4 rows report the $R_{1}$ and $R_{2}$ values in seconds ${ }^{-1}$ as the means. The lower 6 rows report the $P$ values for the group comparisons performed with 1 -way ANOVA and the Hochberg post hoc test.

$\mathrm{b} P<.023$.

Table 3: Associations between the number of GBCA administrations and relaxation rates ${ }^{\mathrm{a}}$

\begin{tabular}{|c|c|c|c|c|c|c|}
\hline & \multicolumn{2}{|c|}{ Linear } & \multicolumn{2}{|c|}{ Mixed } & \multicolumn{2}{|c|}{ Macrocyclic } \\
\hline & Uncorrected & Corrected $^{\mathrm{b}}$ & Uncorrected & Corrected $^{c}$ & Uncorrected & Corrected $^{\mathrm{b}}$ \\
\hline $\mathrm{DN} \mathrm{R}_{1}$ & $0.52, P=.002^{d}$ & $0.50, P=.005^{d}$ & $0.57, P<.001^{d}$ & $0.54, P<.001^{\mathrm{d}}$ & $0.23, P=.19$ & $0.24, P=.16$ \\
\hline $\mathrm{DNR}_{2}$ & $0.14, P=.45$ & $0.04, P=.83$ & $0.30, P=.006^{\mathrm{d}}$ & $0.17, P=.14$ & $0.04, P=.82$ & $0.07, P=.64$ \\
\hline $\mathrm{GPR}_{1}$ & $0.51, P=.004^{\mathrm{d}}$ & $0.50, P=.009^{d}$ & $0.43, P<.001^{\mathrm{d}}$ & $0.37, P=.002^{d}$ & $0.16, P=.36$ & $0.17, P=.33$ \\
\hline $\mathrm{GPR}_{2}$ & $0.28, P=.14$ & $0.26, P=.19$ & $0.43, P<.001^{d}$ & $0.42, P<.001^{d}$ & $0.29, P=.10$ & $0.29, P=.10$ \\
\hline $\mathrm{CNR}_{1}$ & $0.41, P=.020^{d}$ & $0.33, P=.07$ & $0.45, P<.001^{\mathrm{d}}$ & $0.39, P=.001^{\mathrm{d}}$ & $0.24, P=.16$ & $0.27, P=.11$ \\
\hline $\mathrm{CNR}_{2}$ & $0.21, P=.25$ & $0.06, P=.72$ & $0.50, P<.001^{\mathrm{d}}$ & $0.36, P<.001^{d}$ & $0.36, P=.032$ & $0.40, P=.006^{\mathrm{d}}$ \\
\hline Thalamus $R_{1}$ & $0.23, P=.21$ & $0.24, P=.22$ & $0.39, P=.001^{\mathrm{d}}$ & $0.41, P<.001^{d}$ & $0.28, P=.10$ & $0.27, P=.12$ \\
\hline Thalamus $R_{2}$ & $-0.2, P=.26$ & $0.15, P=.44$ & $0.11, P=.32$ & $0.16, P=.17$ & $0.10, P=.59$ & $0.08, P=.65$ \\
\hline
\end{tabular}

${ }^{a}$ All association results are given as $\beta$ coefficients. The healthy controls (GBCA administrations: $n=0$ ) are included in regression analyses for each group.

${ }^{b}$ Corrected for age.

${ }^{c}$ Corrected for age and number of macrocyclic GBCA administrations.

$\mathrm{d} p<.023$.

with a higher $\mathrm{R}_{1}$ in the $\mathrm{DN}, \mathrm{GP}$, and $\mathrm{CN}$. The results for the $\mathrm{DN}$ and GP remained significant after correcting for the patient's age. In the mixed group, the number of linear GBCA administrations was associated with higher $\mathrm{R}_{1}$ in all ROIs, and the results remained significant after correction for the patient's age and the number of macrocyclic GBCA administrations. In the macrocyclic group, no significant associations were found between the number of macrocyclic GBCA administrations and $\mathrm{R}_{1}$. The associations of GBCA administrations with relaxation values are shown in Fig 1.

\section{Associations of GBCA Administrations with $R_{\mathbf{2}}$ Values}

In the linear group, no associations were found between the number of GBCA administrations and $\mathrm{R}_{2}$. In the mixed group, a higher number of linear GBCA administrations was associated with higher $\mathrm{R}_{2}$ in the DN, GP, and $\mathrm{CN}$. The results for the GP and $\mathrm{CN}$ remained significant after correcting for the patient's age and the number of macrocyclic GBCA administrations. In the macrocyclic group, there was a trend toward higher $\mathrm{R}_{2}$ in the $\mathrm{CN}$, which became significant after correction for the patient's age. For the other ROIs, no significant associations were found.

\section{Associations with Cognitive and Physical Disabilities}

In terms of information-processing speed (SDMT), there were few patients with data in the linear group, explaining why only the mixed and macrocyclic groups were studied. A detailed presentation of all regression results with cognitive tests for the mixed group can be found in Table 4 . In the mixed group, there were several significant results and trends between higher relaxation rates and lower information-processing speed. Most notably, higher $\mathrm{R}_{1}$ and $\mathrm{R}_{2}$ in the thalamus were associated lower processing speeds both before and after correction for the MS disease duration, lesion volume, and brain parenchymal fraction. Higher $\mathrm{R}_{2}$ in the DN was also associated with lower SDMT scores before and after these corrections. In the macrocyclic group, no associations were found between $\mathrm{R}_{1}$ and $\mathrm{R}_{2}$ values and information-processing speed $(P=.14-.94)$.

In terms of verbal fluency, there were few patients with data in the linear $(n=4)$ and macrocyclic $(n=6)$ groups, explaining why only the mixed group was studied. In the mixed group, higher $\mathrm{R}_{1}$ in the $\mathrm{CN}$ and thalamus was associated with lower verbal fluency both before and after correction for MS disease duration, lesion volume, and brain parenchymal fraction. A similar trend was found for $\mathrm{R}_{2}$ in the $\mathrm{CN}$ and $\mathrm{GP}$, which, after corrections, became statistically significant. No association was found between verbal fluency and relaxation values in the other brain structures. Figure 2 illustrates the associations of $\mathrm{R}_{1}$ and $\mathrm{R}_{2}$ with information-processing speed and verbal fluency in the mixed group.

No significant associations were found between fatigue or EDSS scores and $R_{1}$ and $R_{2}$ values for any structure (data not shown).

\section{DISCUSSION}

In this study, we applied multiparametric MR imaging in patients with MS and healthy controls to quantitatively study changes in relaxation values and their association with GBCA administration. We have shown that our method has good repeatability and was able to detect relaxation abnormalities associated with GBCA 

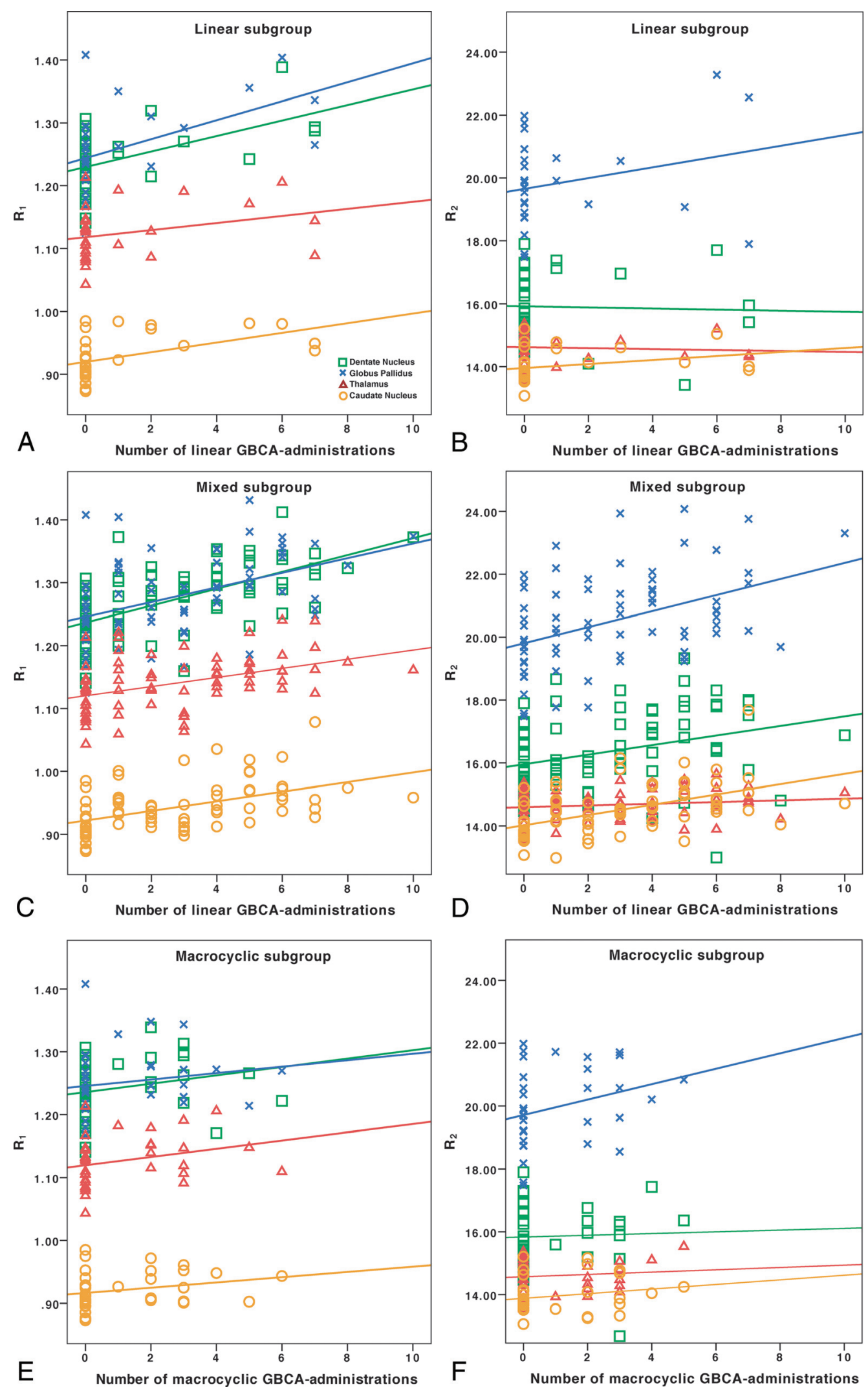

FIG 1. Associations of $R_{1}$ and $R_{2}$ with the number of GBCA administrations. In the linear group, the number of linear GBCA administrations was associated with higher $R_{1}(A)$, but not $R_{2}(B)$. In the mixed group, the number of linear $G B C A$ administrations was associated with both higher $R_{1}(C)$ and $\mathrm{R}_{2}(D)$. The healthy controls, who had not received any GBCA administrations, are represented in each group and included in the regression analyses. The full regression analysis results are reported in Table 3. In the macrocyclic group, no significant associations were found with the number of macrocyclic GBCA administrations $(E$ and $F$. The dentate nucleus is represented by green squares; the globus pallidus, by blue $x$ 's; the caudate nucleus, by orange circles; and the thalamus, by red triangles with corresponding linear regression lines. The $y$-axis presents the relaxation rates $\left(R_{1}\right.$ and $\left.R_{2}\right)$ in seconds ${ }^{-1}$. 
Table 4: Associations between relaxation rates and cognitive tests for the mixed group ${ }^{a}$

\begin{tabular}{|c|c|c|c|c|}
\hline & \multicolumn{2}{|c|}{$\operatorname{SDMT}(n=36)$} & \multicolumn{2}{|c|}{ Verbal Fluency Test $(n=17)$} \\
\hline & Uncorrected & Corrected $^{\mathrm{b}}$ & Uncorrected & Corrected $^{\mathrm{b}}$ \\
\hline $\mathrm{DNR}_{1}$ & $-0.34, P=.04$ & $-0.35, P=.028$ & $-0.38, P=.18$ & $-0.36, P=.13$ \\
\hline $\mathrm{DNR}_{2}$ & $-0.41, P=.012^{c}$ & $-0.37, P=.014^{c}$ & $-0.06, P=.82$ & $-0.04, P=.87$ \\
\hline $\mathrm{GPR}_{1}$ & $-0.32, P=.07$ & $-0.29, P=.13$ & $-0.43, P=.16$ & $-0.31, P=.26$ \\
\hline $\mathrm{GPR}_{2}$ & $-0.18, P=.32$ & $-0.12, P=.51$ & $-0.37, P=.23$ & $-0.64, P=.003^{c}$ \\
\hline $\mathrm{CNR}_{1}$ & $-0.46, P=.005^{c}$ & $-0.34, P=.05$ & $-0.63, P=.009^{c}$ & $-0.68, P=.003^{c}$ \\
\hline $\mathrm{CNR}_{2}$ & $-0.39, P=.018^{c}$ & $-0.21, P=.29$ & $-0.52, P=.04$ & $-0.89, P=.006^{c}$ \\
\hline Thalamus $R_{1}$ & $-0.45, P=.005^{c}$ & $-0.38, P=.014^{c}$ & $-0.59, P=.017^{c}$ & $-0.50, P=.020^{C}$ \\
\hline Thalamus $R_{2}$ & $-0.42, P=.011^{c}$ & $-0.45, P=.002^{c}$ & $-0.44, P=.09$ & $-0.34, P=.10$ \\
\hline
\end{tabular}

${ }^{a}$ All association results are given as $\beta$ coefficients.

${ }^{b}$ Corrected for disease duration, lesion volume, and brain parenchymal fraction.

${ }^{c} p<.023$.
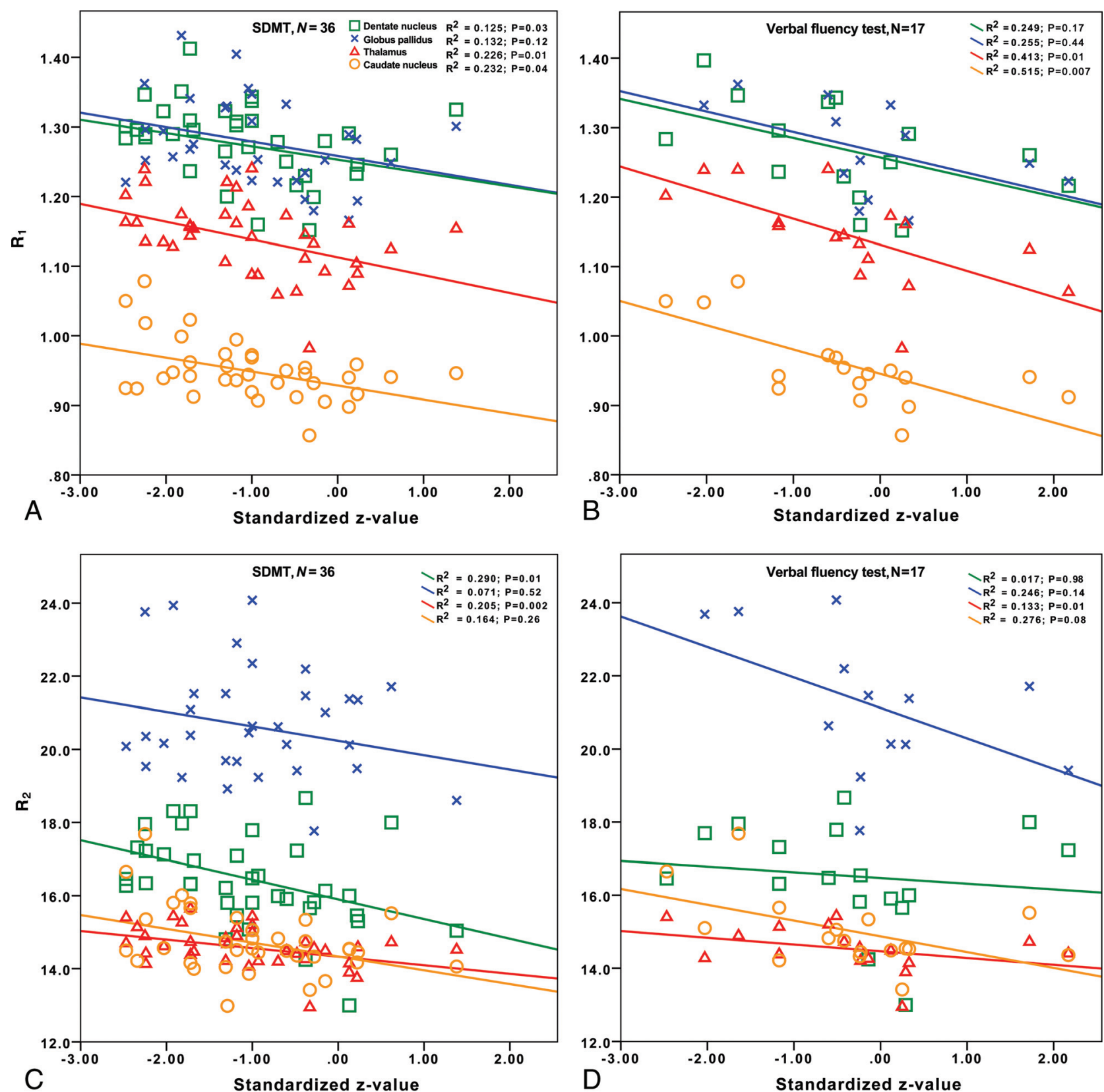

FIG 2. Associations of $R_{1}(A$ and $B)$ and $R_{2}(C$ and $D)$ with information-processing speed and verbal fluency in the mixed group. Please refer to Table 4 for the regression analyses corresponding to this figure. 
administrations not only in $\mathrm{R}_{1}$ but also in $\mathrm{R}_{2}$. We have also provided tentative results regarding associations between relaxation values and cognitive performance in MS.

We found that patients with MS receiving linear GBCAs had higher $\mathrm{R}_{1}$ in the $\mathrm{DN}, \mathrm{GP}$, and $\mathrm{CN}$ (and in the mixed MS group, also the thalamus) compared with healthy controls and that the number of administrations of linear, but not macrocyclic, GBCAs was associated with higher $\mathrm{R}_{1}$ in all studied brain regions, suggesting a dose-dependent association. With regard to the high $\mathrm{R}_{1}$ in at least the DN, increased T1 signal in the DN has previously been associated with MS progression ${ }^{29}$ and could thus be partly due to iron deposits related to neurodegeneration. ${ }^{30}$

Furthermore, the $\mathrm{R}_{2}$ in the GP and $\mathrm{CN}$ was also higher in the mixed group than in healthy controls, a finding indicating that $\mathrm{T} 2$ relaxation is also affected by gadolinium retention. It is also known that chelates that bind to macromolecules cause higher relaxivity. ${ }^{12}$ It is, however, unclear how much the transversal relaxation is affected by retained gadolinium that is bound to different macromolecules or retained in other species (rather than chelated in GBCAs). ${ }^{12}$ This problem highlights the need to also study $\mathrm{T} 2$ effects in the context of gadolinium retention.

The European Medicines Agency has recently decided to suspend the use of several linear types of GBCA, while the US Food and Drug Administration instead merely recommended more precautionary use, particularly of linear GBCAs. ${ }^{13,14}$ In line with the results of most previous studies, we found no associations between the number of administrations of the macrocyclic agent gadoterate meglumine (Dotarem) and the relaxation rates. Macrocyclic agents have histopathologically been shown to be retained to a lower degree than linear GBCAs and have previously shown weaker or no associations in imaging studies. $2,3,11,31,32$ However, the lack of significant associations within the macrocyclic group might also be caused by the small number of patients $(n=15)$ and the relatively few administrations of macrocyclic GBCAs (median, 3) in the current study. Meanwhile, significant associations were found in the linear group, which was even smaller $(n=11)$, illustrating that the methodology used in this study is sensitive in picking up signal changes related to previous GBCA administrations and that the lack of significant results in the macrocyclic group may indeed reflect differences in the degree of retention between linear and macrocyclic GBCAs.

A few industry-sponsored studies on rodents have shown that retained gadolinium from gadodiamide has a time-dependent partial clearance from the brain of healthy rats, which leads to a decrease in $\mathrm{T} 1$ intensity, especially during the first month after the GBCA administration. ${ }^{2,33}$ One of these animal studies also showed, by using histopathologic measurements in the DN, a higher degree of gadolinium clearance for gadoterate meglumine compared with gadodiamide. ${ }^{2}$ However, the current finding of remaining gadolinium retention after the initial washout period is in line with a previous study in which high $\mathrm{T} 1$ hyperintensities on conventional MR imaging remained even 9 years after the last administrations of linear GBCA. ${ }^{17}$ If there is a continued clearance of gadolinium with time, it would have been unfavorable to the macrocyclic GBCAs in our study because there was a shorter time since the last GBCA administration in the macrocyclic group (median, 8 months) in comparison with the mixed (median, 12 months) and linear groups (median, 52 months).

In line with a previous study in which a separate cohort of patients with MS was studied retrospectively with semiquantitative T1-weighted intensity ratios, ${ }^{17}$ significant associations were found in the current study between detected MR imaging changes and lower verbal fluency performance. Furthermore, in this study, we also found associations between lower informationprocessing speed and higher relaxation rates in the DN and thalamus, with similar tendencies also in the GP and CN. Nevertheless, the associations between relaxation rates and cognitive functioning must be interpreted with caution because the MS pathology may confound the results, and although we corrected for the MS disease progression, brain parenchymal fraction, and lesion volume it might not have been sufficient to counteract such confounds. For example, MS is a disease with varying lesion topography (including cortical lesions), numerous treatment regimens, and differences in cognitive reserves among patients, which might also need to be taken into account. ${ }^{34,35}$

Information-processing speed is also one of the most affected cognitive domains in MS, making it even harder to conclude any causality to GBCA exposure. ${ }^{34}$ Designing prospective studies exploring such associations should, therefore, focus on other patient groups exposed to multiple GBCA administrations, with fewer confounders related to the clinical outcome variables, such as in a previous study performed in patients with Crohn disease. ${ }^{36}$ In line with a recent study, ${ }^{37}$ we did not find any association between gadolinium exposure and physical disability in MS. This suggests that future studies should continue to focus on cognitive aspects.

Limitations of the study are mainly related to the results of the MS cohort possibly being confounded by MS itself. The investigative possibilities of the study are also limited by its cross-sectional and noninterventional design, in which the stratification of the groups (based on the type of administered GBCA) is not randomized. There were also relatively few patients who had undergone neuropsychological testing. There are, however, also strengths to the study. By applying a quantitative approach with the same MR imaging scanner, we avoided methodologic limitations of many previous studies with reliance on conventional MR imaging and semiquantitative signal intensity ratios, which may have been due to several risks for confounders such as the diversity of MR imaging parameters and equipment as well as the need for a reference region, which may, in itself, be affected by gadolinium retention. ${ }^{9}$

\section{CONCLUSIONS}

$\mathrm{T} 1$ and $\mathrm{T} 2$ relaxometry can be used to robustly and quantitively study MR imaging changes related to administration of GBCAs, removing the need for a reference region that may, in itself, be confounded by retained gadolinium. The number of linear, but not macrocyclic, GBCA administrations was associated with high $\mathrm{R}_{1}$ in all investigated brain structures and $\mathrm{R}_{2}$ in the GP and $\mathrm{CN}$ in MS. Higher relaxivity in patients with MS receiving linear GBCAs was associated with a lower information-processing speed and verbal fluency in some brain regions, but not with motor function or fatigue. These findings must, however, be interpreted with care because they may be confounded by cognitive decline caused by 
MS. The findings do, however, encourage future studies to further explore possible negative clinical effects of retained gadolinium, especially in regard to cognitive domains and exposure to linear GBCAs.

Disclosures: Yngve Forslin—RELATED: Grant: Karolinska Institutet and Stockholm County Council ALF grant.* Juha Martola-UNRELATED: Payment for Lecture: Santen. Sten Fredrikson-UNRELATED: Payment for Lectures Including Service on Speakers Bureaus: Merck, Novartis, Roche, Sanofi. Tobias Granberg-RELATED: Grant: ALF grant from Stockholm City Council and postdoctoral research fellowship from Swedish Society for Medical Research*; UNRELATED: Employment: Karolinska University Hospital. *Money paid to institution.

\section{REFERENCES}

1. Kanda $\mathrm{T}$, Ishii $\mathrm{K}$, Kawaguchi $\mathrm{H}$, et al. High signal intensity in the dentate nucleus and globus pallidus on unenhanced T1-weighted MR images: relationship with increasing cumulative dose of a gadolinium-based contrast material. Radiology 2014;270:834-41 CrossRef Medline

2. Robert P, Fingerhut S, Factor C, et al. One-year retention of gadolinium in the brain: comparison of gadodiamide and gadoterate meglumine in a rodent model. Radiology 2018;288:424-33 CrossRef Medline

3. Radbruch A, Weberling LD, Kieslich PJ, et al. Gadolinium retention in the dentate nucleus and globus pallidus is dependent on the class of contrast agent. Radiology 2015;275:783-91 CrossRef Medline

4. Jaulent P, Hannoun S, Kocevar G, et al. Weekly enhanced T1weighted MRI with gadobutrol injections in patients with MS: is there a signal intensity increase in the dentate nucleus and the globus pallidus? Eur J Radiol 2018;105:204-08 CrossRef Medline

5. Bjørnerud A, Vatnehol SA, Larsson C, et al. Signal enhancement of the dentate nucleus at unenhanced MR imaging after very high cumulative doses of the macrocyclic gadolinium-based contrast agent gadobutrol: an observational study. Radiology 2017;285:434-44 CrossRef Medline

6. Rossi Espagnet MC, Bernardi B, Pasquini L, et al. Signal intensity at unenhanced T1-weighted magnetic resonance in the globus pallidus and dentate nucleus after serial administrations of a macrocyclic gadolinium-based contrast agent in children. Pediatr Radiol 2017;47:1345-52 CrossRef Medline

7. Splendiani A, Perri M, Marsecano C, et al. Effects of serial macrocyclic-based contrast materials gadoterate meglumine and gadobutrol administrations on gadolinium-related dentate nuclei signal increases in unenhanced T1-weighted brain: a retrospective study in 158 multiple sclerosis (MS) patients. Radiol Med 2018;123: 125-34 CrossRef Medline

8. Radbruch A, Quattrocchi CC. Interpreting signal-intensity ratios without visible $\mathrm{T} 1$ hyperintensities in clinical gadolinium retention studies. Pediatr Radiol 2017;47:1688-89 CrossRef Medline

9. McDonald RJ, McDonald JS, Kallmes DF, et al. Intracranial gadolinium deposition after contrast-enhanced MR imaging. Radiology 2015;275:772-82 CrossRef Medline

10. Tedeschi E, Palma G, Canna A, et al. In vivo dentate nucleus MRI relaxometry correlates with previous administration of gadolinium-based contrast agents. Eur Radiol 2016;26:4577-84 CrossRef Medline

11. Müller A, Jurcoane A, Mädler B, et al. Brain relaxometry after macrocyclic Gd-based contrast agent. Clin Neuroradiol 2017;27:459-68 CrossRef Medline

12. Rohrer M, Bauer H, Mintorovitch J, et al. Comparison of magnetic properties of MRI contrast media solutions at different magnetic field strengths. Invest Radiol 2005;40:715-24 CrossRef Medline

13. European Medicines Agency. EMA's final opinion confirms restrictions on use of linear gadolinium agents in body scans. 625317. July 21, 2017. https://www.ema.europa.eu/en/news/emas-final-opinionconfirms-restrictions-use-linear-gadolinium-agents-bodyscans. Accessed January 29, 2019
14. US Food and Drug Administration. FDA Drug Safety Communication: FDA warns that gadolinium-based contrast agents (GBCAs) are retained in the body; requires new class warnings. 2017. https://www. fda.gov/drugs/drug-safety-and-availability/fda-drug-safetycommunication-fda-warns-gadolinium-based-contrast-agentsgbcas-are-retained-body. Accessed February 15, 2019

15. Welk B, McArthur E, Morrow SA, et al. Association between gadolinium contrast exposure and the risk of parkinsonism. JAMA 2016; 316:96-98 CrossRef Medline

16. $\mathrm{McD}$ onald RJ. No evidence gadolinium causes neurologic harm. RSNA 2017 Daily Bulletin. December 1, 2017. https://rsna2017.rsna. org/dailybulletin/index.cfm?pg=17fri10. Accessed September 5, 2018

17. Forslin Y, Shams S, Hashim F, et al. Retention of gadolinium-based contrast agents in multiple sclerosis: retrospective analysis of an 18-year longitudinal study. AJNR Am J Neuroradiol 2017;38:1311-16 CrossRef Medline

18. Vågberg M, Axelsson M, Birgander R, et al. Guidelines for the use of magnetic resonance imaging in diagnosing and monitoring the treatment of multiple sclerosis: recommendations of the Swedish Multiple Sclerosis Association and the Swedish Neuroradiological Society. Acta Neurol Scand 2017;135:17-24 CrossRef Medline

19. Warntjes JB, Leinhard OD, West J, et al. Rapid magnetic resonance quantification on the brain: optimization for clinical usage. Magn Reson Med 2008;60:320-29 CrossRef Medline

20. Mangeat G, Ouellette R, Warntjes JBM, et al. Accuracy and precision of Synthetic MRI. In: Proceedings of the Joint Meeting of the International Society for Magnetic Resonance in Medicine and the European Society for Magnetic Resonance in Medicine and Biology, Paris, France. June 16-21, 2018

21. Vågberg M, Lindqvist T, Ambarki K, et al. Automated determination of brain parenchymal fraction in multiple sclerosis. AJNR Am J Neuroradiol 2013;34:498-504 CrossRef Medline

22. Granberg T, Uppman M, Hashim F, et al. Clinical feasibility of Synthetic MRI in multiple sclerosis: a diagnostic and volumetric validation study. AJNR Am J Neuroradiol 2016;37:1023-29 CrossRef Medline

23. Schmidt P, Gaser C, Arsic M, et al. An automated tool for detection of FLAIR-hyperintense white-matter lesions in multiple sclerosis. Neuroimage 2012;59:3774-83 CrossRef Medline

24. Ashburner J, Friston KJ. Unified segmentation. Neuroimage 2005;26: 839-51 CrossRef Medline

25. Errante Y, Cirimele V, Mallio CA, et al. Progressive increase of T1 signal intensity of the dentate nucleus on unenhanced magnetic resonance images is associated with cumulative doses of intravenously administered gadodiamide in patients with normal renal function, suggesting dechelation. Invest Radiol 2014;49:685-90 CrossRef Medline

26. Yushkevich PA, Piven J, Hazlett HC, et al. User-guided 3D active contour segmentation of anatomical structures: significantly improved efficiency and reliability. Neuroimage 2006;31:1116-28 CrossRef Medline

27. Jenkinson M, Beckmann CF, Behrens TE, et al. FSL. Neuroimage 2012;62:782-90 CrossRef Medline

28. Benjamini Y, Hochberg Y. Controlling the false discovery rate: a practical and powerful approach to multiple testing. Journal of the Royal Statistical Society. Series B: Methodological 1995;57:289-300 CrossRef

29. Roccatagliata L, Vuolo L, Bonzano L, et al. Multiple sclerosis: hyperintense dentate nucleus on unenhanced T1-weighted MR images is associated with the secondary progressive subtype. Radiology 2009; 251:503-10 CrossRef Medline

30. Bond KM, Brinjikji W, Eckel LJ, et al. Dentate update: imaging features of entities that affect the dentate nucleus. AJNR Am J Neuroradiol 2017;38:1467-74 CrossRef Medline

31. Robert P, Lehericy S, Grand S, et al. T1-weighted hypersignal in the deep cerebellar nuclei after repeated administrations of gadolinium-based contrast agents in healthy rats: difference between linear 
and macrocyclic agents. Invest Radiol 2015;50:473-80 CrossRef Medline

32. Jost G, Frenzel T, Boyken J, et al. Long-term excretion of gadolinium-based contrast agents: linear versus macrocyclic agents in an experimental rat model. Radiology 2019;290:340-48 CrossRef Medline

33. Smith AP, Marino M, Roberts J, et al. Clearance of gadolinium from the brain with no pathologic effect after repeated administration of gadodiamide in healthy rats: an analytical and histologic study. $R a-$ diology 2017;282:743-51 CrossRef Medline

34. Chiaravalloti ND, DeLuca J. Cognitive impairment in multiple sclerosis. Lancet Neurol 2008;7:1139-51 CrossRef Medline
35. Thompson AJ, Banwell BL, Barkhof F, et al. Diagnosis of multiple sclerosis: 2017 revisions of the McDonald criteria. Lancet Neurol 2018;17:162-73 CrossRef Medline

36. Mallio CA, Piervincenzi C, Gianolio E, et al. Absence of dentate nucleus resting-state functional connectivity changes in nonneurological patients with gadolinium-related hyperintensity on T1weighted images. J Magn Reson Imaging 2019 Jan 25. [Epub ahead of print] CrossRef Medline

37. Cocozza S, Pontillo G, Lanzillo R, et al. MRI features suggestive of gadolinium retention do not correlate with Expanded Disability Status Scale worsening in multiple sclerosis. Neuroradiology 2019; 61:155-62 CrossRef Medline 